\title{
Haemoperitonium : A Rare Presentation of Choriocarcinoma
}

\author{
Adiba Malik ${ }^{{ }^{*}}$ \\ Shireen Akter Khanom ${ }^{2}$
}

'Department of Obstetrics \& Gynaecology Chattogram Maa-O-Shishu Hospital Medical College Chattogram, Bangladesh.

${ }^{2}$ Department of Obstetrics \& Gynaecology Chittagong Medical College

Chattogram, Bangladesh.

\section{*Correspondence to:}

\section{Dr. Adiba Malik}

Associate Professor

Department of Obstetrics \& Gynaecology Chattogram Maa-O-Shishu Hospital Medical College Chattogram, Bangladesh.

Mobile : +8801817 732698

Email :dradibamalik@yahoo.com

Date of Submission : $\quad 29.02 .2020$

Date of Acceptance : $\quad 15.06 .2020$

www.banglajol.info/index.php/CMOSHMCJ

\begin{abstract}
Gestational choriocarcinoma is one of the most malignant form of group of tumors with almost $100 \%$ cure rate, developes secondary to pregnancy (Normal or ectopic) following hydatidiform mole, miscarriage or even after child birth. This starts within the uterus in the placenta but a tendency to metastasize to other parts of the body. Indicative signs are unexplained heavy and irregular uterine bleeding, raised serum $\beta$ hCG, non normalization of $\beta$ hCG following abortion or delivery and if it spreads, signs of metastasis. Our study presents a case of Choriocarcinoma presented with haemoperitonium, who was treated successfully with surgical and medical management. A 25 years lady Para 1+0, age of 3 years, normal delivery had an emergency admission for 2 months amenorrhoea with severe lower abdominal pain, slight vaginal bleeding suspected clinically as ruptured ectopic pregnancy. Ultrasonography suggest Invasive mole with huge peritoneal collection. After sending serum $\beta$ hCG she was treated surgically and per operative findings were in favour of Choriocarcinoma which was confirmed by histopathological reports. Serum $\beta$ hCG was raised and she was treated with combination (MAC protocol) chemotherapy.
\end{abstract}

Key words : Haemoperitonium; Miscarriage; Chriocarcinoma, Histopathology.

\section{INTRODUCTION}

Choriocarcinoma also known as trophoblastic malignancy is a rare form of cancer with almost $100 \%$ cure rate. The incidence of choriocarcinoma is estimated to be 0.133 per 100000 woman years ${ }^{1}$. Majority of cases of choriocarcinoma arises due to malignant transformation of complete mole, although it has been reported following abortion, normal pregnancy, ectopic pregnancy or following child birth ${ }^{2}$. It is known to occur 1 in 5333 of tubal pregnancy and 1 in 1.6 million of normal intrauterine pregnancy ${ }^{3}$. It can develop anytime between 5 weeks and 15 years after geststion or even after menopause ${ }^{4}$. This highly malignant tumour usually starts in the uterus but can rapidly spread to other parts of body. Approximately $30 \%$ cases have metastatic disease at the time of diagnosis. Lungs (80\%) are the most common sites of metastasis followed by vagina (30\%) liver (10\%) and involving brain in $28 \%$ of patients $^{5}$. So before starting treatment of molar pregnancy $\beta$ hCG (Human Chorionic Gonadotrophin) and chest X Ray should be done. The leading symptom is heavy and irregular uterine hemorrhage sooner or later expulsion of mole or any recognized form of pregnancy ${ }^{6}$. The other symptoms are vaginal bleeding, anemia, hyperemesis, hyperthyroidism, uterine and ovarian enlargement and symptoms and signs of metastasis. Because of these variable presentations, it is a diagnostic challenge. Although choriocarcinoma is very aggressive in nature, its cure rate is very high because it is extremely sensitive to chemotheraputic agents. So early diagnosis and prompt initiation of treatment is a well known determinant of prognosis of choriocarcinoma. The cause of gestational trophoblastic disease is claimed to be genetic in origin. No environmental etiological factor has been implicated apart from deficient Vitamin A precursor carotene in diet $^{7}$. 


\section{CASE REPORT}

A 25 years lady para $1+0$ had age of 3 years, normal delivery, took an emergency admission due to 8 weeks amenorrhoea with severe lower abdominal pain and scanty vaginal bleeding and gives history of excessive vomiting and urinary pregnancy test positive at home one week prior to her admission. She was moderately anaemic, abdomen was distended and uterus was palpable about 14 weeks size. Nothing was abnormal on vaginal examination, anterior fornice was free. All the features were in favour of ruptured ectopic pregnancy only exception was hyperemesis and enlarged uterus. Emergency Ultrasonography was done which suggested Invasive mole with peritoneal collection then after counselling, informed written consent was taking blood sent for hCG, grouping and crossmatching, emergency laparotomy was done. On opening it was full of blood, uterus was about 14 weeks size, firm with multiple hemorrhagic and necrotic spots with bleeding from serosal surfaces, total abdominal hysterectomy was done, ovaries and rest of the pelvic organs were normal. On cut section uterus was congested with polypoid growth with hemorrhage and necrotic spots with a clinical suspicion of choriocarcinoma. Histopathological reports confirms the diagnosis of choriocarcinoma. $\beta$ hCG level was raised $(12920 \mathrm{mIU} / \mathrm{ml})$ which is a bit lower range for Choriocarcinoma ${ }^{6}$. Post operative X Ray chest was done and it was normal. with the consultation of Oncologist she completed the course of combination chemotherpy. The patient was withstand well with surgery and had 3 successive normal levels of serum $\beta$ hCG.

\section{DISCUSSION}

choriocarcinoma almost always develops with or follows some form of recognized pregnancy, most often following Hytatidiform mole but may follow abortion, ectopic pregnancy, normal pregnancy or even after delivery.
So before starting treatment of Hydatidiform mole or in case of excessive undiagnosed bleeding following any recognized pregnancy, serum $\beta$ hCG, $X$ Ray chest, liver enyzmes, CBC should be done $e^{7,8}$. The clinical presentation of Choriocarcinoma can be from disease locally in the uterus or from distant metastasis that can cause a wide variety of symptoms ${ }^{9}$. The outcome of choriocarcinoma are related to staging and risk assessment (Low risk, High risk) and not to the antecedent pregnancy ${ }^{10}$. In our study the patient presented with the symptoms and signs mimicking ruptured ectopic pregnancy, the only finding in favour of Choriocarcinoma was hyperemesis and enlarged uterus. The clinical condition of the patient and ultrasonographic diagnosis of invasive mole with haemoperitonium demanded emergency laparotomy and the per operative findings were in favour of Choriocarcinoma, which was confirmed by raised serum $\beta$ hCG and histopathology report. The Choriocarcinoma is functional tumor and usually secretes $\beta$ hCG in large quantity and this causes lutenization with cyst formation in ovaries. Occasionally $\beta$ hCG level may be low when it is composed of non secretory elements as in our patient ${ }^{6}$. Non gestational choriocarcinoma also occur and it is resistant to therapy ${ }^{11}$.

\section{CONCLUSION}

A complete knowledge regarding variable clinical presentation of Choriocarcinoma helps in early diagnosis and decrease the adverse outcome of this rare and highly malignant but curable malignancy. So every pregnancy following missed period with any excessive and abnormal uterine bleeding or any abnormal presentation should be evaluated by serum $\beta$ hCG and ultrasonography and histopathology.

\section{DISCLOSURE}

Both the authors declared no competing interest.

\section{REFERENCES}

1. Lee S M et al. A successfully treated case of tubal choriocarcinoma coexistent with viable ectopic pregnancy. Gynecol. Oncol. 2005; 97(2):671-673.

2. S Meddeb et al. Unusual gestational choriocarcinoma arising in an interstitial pregnancy. Int J Surg Case Rep. 2004;5:787- 788.

3. M A Rettenmaier et al. Gestational Choriocarcinoma in the fallopian tube. J obstet Gynecol. 2013;33:912-914.

4. A. Hassadia et al. Ectopic gestayional trophoblastic disease a case review J Reprod Med. 2012;57:297-300.

5. R S Berkowitz et al. Gestational trophoblastic disease. 2010;376 (9742):717-729.

6. Jeffcoats Principles of Gynaecology Eighth Edition New Delhi, India: Jaypee BrothersMedical Publishers(P). 2014;155.

7. Kajii T et al. Androgenetic origin of Hydatidiform mole Nature. 1977; 268: 633-645 (PubMed).

8. Soto Wright. The changing clinical presentation of complete molar pregnancy Obstet Gynecol. 1995; 86: 775-779.

9. Dewhurtsts Text book of Obstetrics \& Gynaecology Eighth Edition Wiley Blackwell.D. Keith Edmonds ISBN-13. 2012;68.

10. Nugent et al. Postpartum choriocarcinoma presentation management and survival J Reprod Med. 2006;51(10):819-824.

11. Berkowitz R S. risk factors of complete molar pregnancy from a case controll study Am J Obstet Gynecol. 1985;15;152(8): 1016-1020. 